Algebraic $8 \mathcal{G}$ Geometric $\mathcal{T}_{\text {opology }}$

Volume 1 (2001) 503-518

Published: 22 September 2001

ATG

\title{
Generalized symplectic rational blowdowns
}

\author{
Margaret Symington
}

\begin{abstract}
We prove that the generalized rational blowdown, a surgery on smooth 4-manifolds, can be performed in the symplectic category.
\end{abstract}

AMS Classification 57R17; 57R15, 57M50

Keywords Symplectic surgery, blowdown

\section{Introduction}

Surgery techniques are essential tools for understanding the topology of manifolds. For smooth manifolds the rational blowdown surgery, introduced by Fintushel and Stern, is particularly useful because one can calculate how the Donaldson and Seiberg-Witten invariants change when the surgery is performed [6]. For instance, Fintushel and Stern [6] used it to calculate the Donaldson and Seiberg-Witten invariants of simply connected elliptic surfaces and to construct an interesting family of simply connected smooth 4-manifolds $Y(n)$ not homotopy equivalent to any complex surface. This surgery can also be performed in the symplectic category [12], and thereby helps demonstrate the vastness of the set of symplectic 4-manifolds. In particular, the aforementioned $Y(n)$, as well as an infinite family of exotic K3 surfaces [7] (4-manifolds that are homeomorphic but not diffeomorphic to a degree 4 complex hypersurface in $\mathbb{C} P^{3}$ ), all admit symplectic structures [12].

The rational blowdown surgery amounts to removing a neighborhood of a linear chain of embedded spheres whose boundary is the lens space $L\left(n^{2}, n-1\right), n \geq 2$ and replacing it with a rational ball (manifold with the same rational homology as a ball), also with boundary $L\left(n^{2}, n-1\right)$. This has the effect of reducing the dimension of the second homology of $M$ at the expense of possibly complicating the fundamental group. The surgery gets its name from the well-known process of blowing down a -1 sphere (the case $n=1$ ) in which one replaces a tubular neighborhood of a sphere of self-intersection -1 by a 4 -ball.

In fact, there are other lens spaces that bound rational balls: $L\left(n^{2}, n m-\right.$ 1), $n, m \geq 1$ and relatively prime [3]. Therefore one can define a broader 


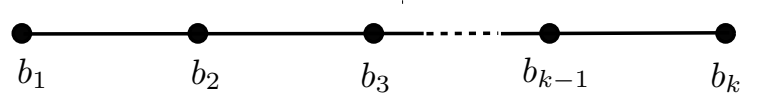

Figure 1: Plumbing diagram for $C_{n, m}$.

class of rational blowdowns, so called generalized rational blowdowns. Park [10] extended Fintushel and Stern's calculations, showing how a generalized rational blowdown affects the Donaldson and Seiberg-Witten invariants of a smooth 4manifold. Here we show that even the generalized rational blowdown can be performed in the symplectic category.

Specifically, given any pair of relatively prime integers $y, x, x \neq 0$, the fraction $\frac{y}{x}$ has a negative continued fraction expansion

$$
b_{1}-\left(\frac{1}{b_{2}-\frac{1}{\cdots-\frac{1}{b_{k}}}}\right)=\frac{y}{x}
$$

which is unique if one assumes that $b_{j} \geq 2$ for all $j \geq 2$. The shorthand for this continued fraction expansion is $\left[b_{1}, b_{2}, \ldots, b_{k}\right]$.

Definition 1.1 For any relatively prime $n \geq 2, m \geq 1$, let $C_{n, m}$ be a closed tubular neighborhood of the union of spheres $\left\{S_{j}\right\}_{j=1, \ldots k}$ in the plumbing of disk bundles represented by the diagram in Figure 1 , where the $b_{j}$ satisfy $\left[b_{1}, b_{2}, \ldots, b_{k}\right]=\frac{n^{2}}{n m-1}$ and $b_{j} \geq 2$ for all $j$.

The spheres in $C_{n, m}$ have the following intersection pattern:

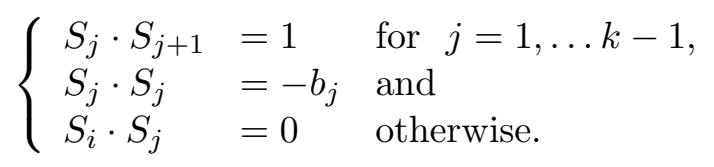

The fact that $S_{j} \cdot S_{j}=-b_{j} \leq-2$ for each $j$ implies that the intersection form of $C_{n, m}$ is negative definite. The boundary of $C_{n, m}$ is the lens space $L\left(n^{2}, n m-1\right)$ which bounds a rational homology ball $B_{n, m}[3,10]$.

Definition 1.2 If there is an embedding $\psi: C_{n, m} \rightarrow M$, then the generalized rational blowdown of $M$ along the spheres $\psi\left(\cup_{i=1}^{k} S_{i}\right)$ is

$$
\widetilde{M}:=\left(M-\psi\left(\cup_{i=1}^{k} S_{i}\right)\right) \cup_{\phi} B_{n, m}
$$

where $\phi$ is an orientation preserving diffeomorphism of a collar neighborhood of the boundary $L\left(n^{2}, n m-1\right)$. 
Theorem 1.3 Suppose $\widetilde{M}=\left(M-\psi\left(\cup_{i=1}^{k} S_{i}\right)\right) \cup_{\phi} B_{n, m}$ is the generalized rational blowdown of a smooth 4-manifold $M$ along spheres $\psi\left(\cup_{i=1}^{k} S_{i}\right)$. If $M$ admits a symplectic structure for which the spheres are symplectic, then the diffeomorphism $\phi$ can be chosen so that $\widetilde{M}$ admits a symplectic structure induced from the symplectic structures on $M$ and $B_{n, m}$.

The essence of the proof, as for the case $m=1$, is in our choice of symplectic models for the spaces $C_{n, m}$ and $B_{n, m}$. By a version of the symplectic neighborhood theorem any neighborhood of symplectic spheres that is diffeomorphic to $C_{n, m}$ has a neighborhood symplectomorphic to a toric model space. (A symplectic manifold is toric if it is equipped with an effective Hamiltonian $T^{n}$ action.)

The new ingredient in this paper is a set of symplectic representatives for the rational balls $B_{n, m}$ for all $m \geq 1$. These representatives are toric near the boundary and can be chosen to "fit" a collar neighborhood of the boundary of $C_{n, m}$. We present the $B_{n, m}$ as the total space of a singular Lagrangian fibration with two types of singular fibers: a one parameter family of circle fibers and one isolated nodal fiber - a sphere with one positive self-intersection. In the language of Hamiltonian integrable systems the singularity of the nodal fiber is a focus-focus singularity. Our nodal fiber is the Lagrangian analog of the singular fibers that appear in Lefschetz fibrations of symplectic 4-manifolds.

Acknowledgments The author thanks Eugene Lerman for suggesting helpful references, in particular the work of Nguyen Tien Zung, and thanks Nguyen Tien Zung in turn for mentioning Vu Ngoc San's work. The author also thanks an anonymous referee who pointed out a minor error.

The author is grateful for the support of an NSF post-doctoral fellowship, DMS9627749.

\section{Background}

Our objective is to control the symplectic structure of collar neighborhoods of the boundaries of the spaces involved in our surgery: $C_{n, m}$ and $B_{n, m}$. We do this by presenting them as the total spaces of singular Lagrangian fibrations. The space $C_{n, m}$ itself and a collar neighborhood of the boundary of $B_{n, m}$ admit singular Lagrangian fibrations equivalent to the fibration defined by the moment map for a Hamiltonian torus action. An important feature of these fibrations is that, at least near the boundary, the base classifies the neighborhood up to fiberwise symplectomorphism (cf. $[2,13]$ ). 
Definition 2.1 A Lagrangian fibration $\pi:\left(M^{2 n}, \omega\right) \rightarrow B^{n}$ is a locally trivial fibration such that $\left.\omega\right|_{\pi^{-1}(b)}=0$ for each $b \in B$ (i.e. such that each fiber is a Lagrangian submanifold).

The Arnold-Liouville theorem guarantees that if the fibers of a Lagrangian fibration are closed (compact, without boundary) and connected then they must be $n$-tori with neighborhoods equipped with canonical coordinates: action-angle coordinates. The local action coordinates supply the base $B$ with an integral affine structure, i.e. an atlas $\Phi_{j}: U_{j} \rightarrow \mathbb{R}^{n}$ with the maps $\left.\Phi_{j} \Phi_{k}^{-1}\right|_{U_{j} \cap U_{k}} \in$ $G L(n, \mathbb{Z})$.

It is easy to see that in dimension 4 (with $n=2$ ) the fibers must be tori: the Lagrangian condition implies the existence of an isomorphism between the normal and tangent bundles defined via the symplectic form; then, since the normal bundle of a fiber must be trivial, we have that Euler characteristic of the tangent bundle is 0 . Because the fiber of a locally trivial fibration of an oriented manifold is orientable, the fiber must be a torus.

We now expand our definition of a Lagrangian fibration to include singular fibers: one parameter families of circle fibers, isolated points and isolated nodal fibers (spheres with one positive transverse intersection). These singular fibrations are examples of Lagrangian fibrations with topologically stable and nondegenerate singularities such as arise in integrable systems [13]. In the spirit of holomorphic fibrations and smooth Lefschetz fibrations, and for simplicity of exposition, we often suppress the word singular. We assume throughout that fibers are connected and that the generic fibers are closed manifolds.

Near the circle and point fibers the fibration is equivalent to one coming from the moment map for a torus action. Therefore, the integral affine structure on the image of the regular fibers, $B_{0} \subset B$, extends to each connected component of the image of the circle fibers. These components meet at the vertices of $B$, the images of the point fibers. The images of nodal fibers are isolated interior points of $B$.

To understand the base $B$ in each of our examples, we view $B$ (or part of it) as a subset of $\mathbb{R}^{2}$. We always assume that $\mathbb{R}^{2}$ is equipped with the integral affine structure coming from the standard lattice generated by the vectors $(1,0)$ and $(0,1)$. It is important to note that there are two different classes of lines in this integral affine space: rational and irrational (as determined by the slope of the line). Indeed, a vector $v$ directed along a line of rational slope has an affine length $\alpha \in \mathbb{R}^{+}$defined by $v=\alpha u$ for a primitive integral vector $u$, while a vector directed along a line of irrational slope does not have a well-defined 
length. By an integral polygon in $\mathbb{R}^{2}$ we mean one whose edges define vectors of the form $\alpha u$ where $\alpha \in \mathbb{R}^{+}$and $u$ is a primitive integral vector, or alternatively, one whose edges all have well-defined affine lengths.

We now review a few facts that facilitate reading the topology of a Lagrangian fibered symplectic 4-manifold $\pi:(M, \omega) \rightarrow U$ from the base $U$ when $U$ coincides with a moment map image. The reader interested in more detail on the topology of a toric symplectic manifold should consult [1]. Throughout our discussion, neighborhood refers to a tubular neighborhood, $\left(p_{1}, p_{2}\right)$ are Euclidean coordinates on $\mathbb{R}^{2}$ and $\left(q_{1}, q_{2}\right)$ are circular coordinates on $T^{2}$.

(1) A simply connected open domain $U \subset \mathbb{R}^{2}$ defines the open symplectic manifold $\left(U \times T^{2}, d p \wedge d q\right)$.

(2) An open neighborhood $U$ of a point in the boundary of a closed half-plane in $\mathbb{R}^{2}$ defines the smooth manifold $S^{1} \times D^{3}$ that is symplectomorphic to a neighborhood of $\left\{\left(z_{1}, z_{2}\right)|0<| z_{1}|<\alpha,| z_{2} \mid=0\right\} \subset\left(\mathbb{C}^{2}, \frac{1}{2 i} d \bar{z} \wedge d z\right)$ for some $\alpha \in \mathbb{R}^{+}$. The symplectic structure $d p \wedge d q$ defined on the preimage of int $U$ extends to the circles that live over the points in $\partial U \cap U$. If the half space is bounded by the line $\left\{\left(p_{1}, p_{2}\right) \mid p_{2}=\frac{m}{n} p_{1}\right\} \subset \mathbb{R}^{2}$ then the circle fibers are quotients $T^{2} /\left(q_{1}, q_{2}\right) \sim\left(q_{1}-m t, q_{2}+n t\right), t \in \mathbb{R}$ of the tori living over points in $U$ with $p_{2}=\frac{m}{n} p_{1}$.

(3) A neighborhood $U$ of a vertex in a convex integral polygon defines a symplectic 4 -ball if and only if the primitive integral vectors $u, v$ that define the directions of the adjacent edges satisfy $|u \times v|=1$. (Here $\times$ is the cross product in $R^{3}$ restricted to $R^{2}$, thus yielding a scaler.) The preimage of the vertex is a point. If $|u \times v|=n \geq 2$ then $U$ defines a neighborhood of an orbifold singularity.

(4) A neighborhood $U$ of an edge $E$ in a convex polygon defines a neighborhood of a sphere. Specifically, consider an edge $\alpha w$, with $\alpha \in \mathbb{R}^{+}$and $w$ a primitive integral vector. Suppose $u, v$ are the primitive integral vectors based at the endpoints of $E$ that define (up to scaling) the left and right adjacent edges. Then the sphere has area $\alpha$ and self-intersection $u \times v$. See Figure 2.

(5) If $U \subset \mathbb{R}^{2}$ defines a toric symplectic manifold, then for any $A \in G L(2, \mathbb{Z})$ and $b \in \mathbb{R}^{2}, A(U)+b$ defines the same symplectic manifold (with a different torus action if $A$ is not the identity). 


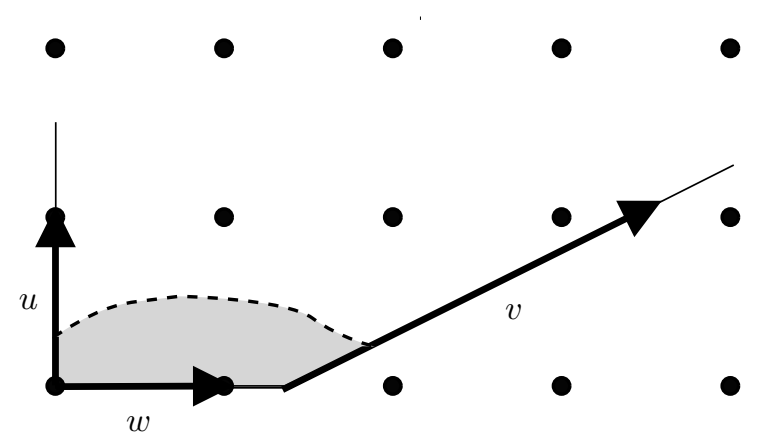

Figure 2: Neighborhood of a sphere of self-intersection -2 and area $\frac{3}{2}$.

\section{$3 \quad$ Symplectic models}

In this section we provide symplectic models for the cone on a lens space, neighborhoods of certain linear chains of spheres, the neighborhood of a nodal fiber, and rational balls. We give the descriptions in terms of diagrams in $\mathbb{R}^{2}$ that correspond to images of moment maps when a global torus action can be defined.

The examples we present here are the building blocks for our constructions and are essential for the proof of Theorem 1.3.

\subsection{Toric models}

Example 3.1 Cone on a lens space $L(n, m)$.

Consider the following subset of $\mathbb{R}^{2}$ :

$$
V_{n, m}=\left\{p_{1} \geq 0\right\} \cap\left\{p_{2} \geq \frac{m}{n} p_{1}\right\} \cap\left\{p_{2}>0\right\}
$$

and the (singular) Lagrangian fibered symplectic manifold $\pi:(M, \omega) \rightarrow V_{n, m}$ it defines. Figure 3 shows $V_{n^{2}, n m-1}$, the case we are interested in.

To see that $M$ is a cone on a lens space, recall that $L(n, m)$ can be decomposed as the union of two solid tori glued together via a map $\phi$ of their boundaries such that $\phi_{*} \mu_{2}=-m \mu_{1}+n \lambda_{1}$ where $\mu_{i}, \lambda_{i}$ are meridinal and longitudinal cycles on the solid torus boundaries.

For any $t>0$, consider the 3-manifold in $M$ that is the preimage of $\left\{p_{2}=t\right\} \cap$ $V_{n, m}$; decompose it as the union of preimages $P_{1} \cup P_{2}$ where $P_{1}$ is the preimage 


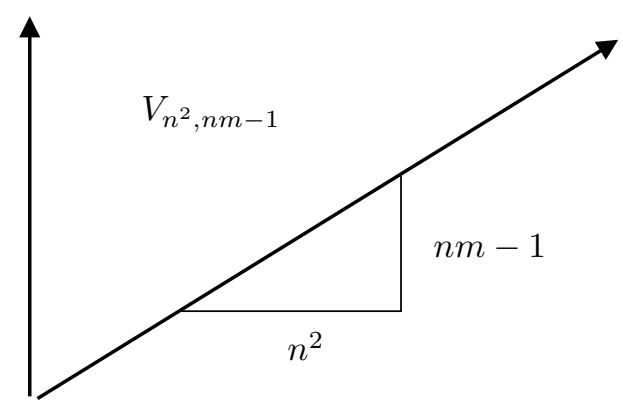

Figure 3: Cone on the lens space $L\left(n^{2}, n m-1\right)$.

of $\left\{p_{1} \leq c t, p_{2}=t\right\} \cap V_{n, m}$ and $P_{2}$ is the preimage of $\left\{p_{1} \geq c t, p_{2}=t\right\} \cap V_{n, m}$ for some $0<c<\frac{n}{m}$. Then $P_{1}, P_{2}$ are a solid tori with meridians whose tangent vectors are $\frac{\partial}{\partial q_{1}}$ and $-m \frac{\partial}{\partial q_{1}}+n \frac{\partial}{\partial q_{2}}$ respectively, thereby showing the 3 -manifold is $L(n, m)$. Letting $t$ vary we get $L(n, m) \times(0, \infty)$.

There was nothing special about the choice of $\left\{p_{2}=t\right\} \cap V_{n, m}$ to define the lens space; we could have used any arc smoothly embedded in $V_{n, m}$ with one endpoint on each of the edges of $V_{n, m}$. However, by choosing an arc $\gamma$ transverse to the vector field $p_{1} \frac{\partial}{\partial p_{1}}+p_{2} \frac{\partial}{\partial p_{2}}$ we get an induced contact structure (completely non-integrable 2-plane field, cf. [5]) on the lens space. This contact structure is defined as the kernel of the 1 -form $\left.\iota_{X} \omega\right|_{\pi^{-1}(\gamma)}$ where $X$ is the unique vector field on $M$ which is given by $p_{1} \frac{\partial}{\partial p_{1}}+p_{2} \frac{\partial}{\partial p_{2}}$ in the local coordinates $(p, q)$ on $\pi^{-1}$ (int $\left.V_{n, m}\right)$. The contact structure is independent of the choice of the transverse $\operatorname{arc} \gamma$.

Example 3.2 Negative definite chains of spheres.

Here we define a neighborhood of a chain of spheres by a neighborhood of the piecewise linear boundary of a domain in $\mathbb{R}^{2}$. See Figure 4 for an example.

Let $\left\{x_{j}\right\}_{j=0}^{k}$ be a set of points in $\mathbb{R}^{2}$ and $\left\{u_{j}\right\}_{j=0}^{k+1}$ a set of primitive integral vectors such that

- $\alpha_{j} u_{j}=x_{j}-x_{j-1}$ with $\alpha_{j} \in \mathbb{R}^{+}$for each $1 \leq j \leq k$,

- $u_{j} \times u_{j+1}=1$ for each $0 \leq j \leq k$, and

- $u_{j+1} \times u_{j-1}=S_{j} \cdot S_{j}$ for each $1 \leq j \leq k$.

Let $X$ be the convex hull of the points $\left\{x_{j}\right\}_{j=0}^{k}$ and all points $x$ such that $x_{0}-x=\alpha u_{0}$ or $x-x_{k}=\alpha u_{k+1}$ for some $\alpha>0$. 


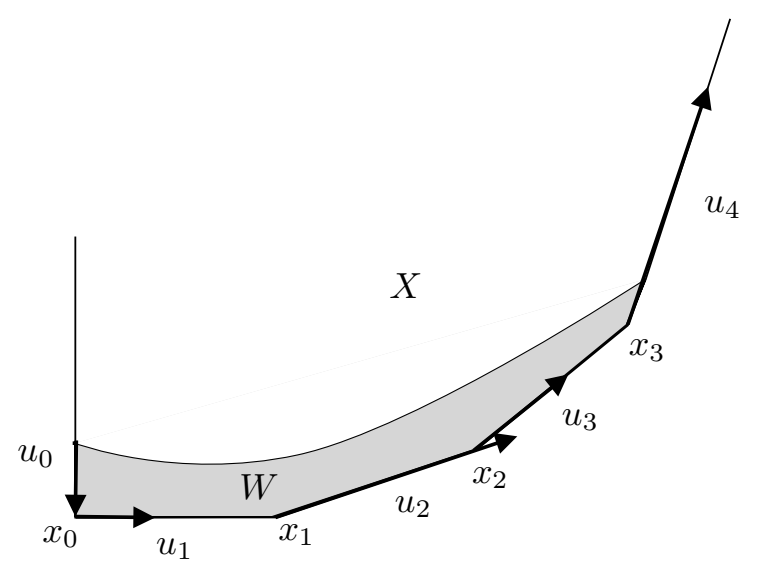

Figure 4: Neighborhood of spheres.

Then $X$ defines a Lagrangian fibered symplectic manifold $(M, \omega) \rightarrow X$ such that each finite edge, defined by the vector $x_{j}-x_{j-1}$ for some $j$, is the image of a sphere $S_{j}$. The area of each sphere $S_{j} \subset M$ is $\alpha_{j}$ and for each $1 \leq j \leq$ $k-1, S_{j}$ intersects $S_{j+1}$ once positively and transversely. The convexity of $V$ corresponds to the negative definiteness of the intersection form of $M$.

Let $W$ be any closed neighborhood in $X$ of the finite edges defined by the $x_{j}$. Then $W$ defines a singular Lagrangian fibration of a closed toric neighborhood of spheres $S_{1}, \ldots S_{k}$ in $M$. (We interpret the points in $\partial W \cap$ int $X$ as the images of tori, not circles.)

A variation of the symplectic neighborhood theorem states that the germ of the neighborhood of a linear chain of spheres is determined up to symplectomorphism by the areas of the spheres and the intersection form. (An explanation of how Moser's method would be applied in this case is provided in [9].) Therefore, given any symplectic manifold $(M, \omega)$ containing a smoothly embedded copy of $C_{n, m}$ as a neighborhood of symplectic spheres, we can choose an $X=X_{n, m}$ and a $W_{n, m} \subset X_{n, m}$ small enough that $W_{n, m}$ defines a Lagrangian fibered symplectic manifold that symplectically embeds in and is diffeomorphic to the embedded copy of $C_{n, m}$. Therefore, we simply assume that $C_{n, m}$ is symplectically embedded in $M$ and Lagrangian fibers over $W_{n, m}$. We also assume, without loss of generality, that the boundary of $C_{n, m}$ has an induced contact structure equivalent to the one described in Example 3.1 when the lens space is $L\left(n^{2}, n m-1\right)$. 


\subsection{Neighborhood of a nodal fiber}

Nodal fibers appear as singular fibers in numerous integrable systems including the spherical pendulum (cf. [4, 14]). As noted by Zung [14], a simple model for a Lagrangian fibered neighborhood of a nodal fiber is a self-plumbing of the zero section of $\left(T^{*} S^{2}, \omega=\operatorname{Re} d z_{1} \wedge d z_{2}\right)$. Indeed, glue a neighborhood of $(0,0) \subset \mathbb{C}^{2}$ to a neighborhood of $(\infty, 0)$ by the symplectomorphism $\left(z_{1}, z_{2}\right) \rightarrow\left(z_{2}^{-1}, z_{1} z_{2}^{2}\right)$. Projecting to $\mathbb{R}^{2}=\mathbb{C}$ by the map $z_{1} z_{2}$ gives the desired Lagrangian fibration.

Lemma 3.3 The germ of a symplectic neighborhood of a Lagrangian nodal fiber is unique up to symplectomorphism.

Proof The lemma follows from the Lagrangian neighborhood theorem by pulling the symplectic structure of a nodal fiber neighborhood back to a neighborhood of the zero section of $T^{*} S^{2}$ via an immersion.

Let $\pi:(N, \omega) \rightarrow B$ be a Lagrangian fibered neighborhood of a nodal fiber with $B$ a disk and $b_{0} \in B$ the image of the nodal fiber. The Arnold-Liouville theorem implies that $B-b_{0}$ is equipped with an integral affine structure. In particular, $T\left(B-b_{0}\right)$ has a flat connection. The topological monodromy

$$
A=\left(\begin{array}{ll}
1 & 1 \\
0 & 1
\end{array}\right)
$$

of the torus fibration over $B-b_{0}$ and the Lagrangian structure of the fibration forces the same monodromy in the induced flat connection on $T\left(B-b_{0}\right)$. Therefore no embedding of $B$ into $\mathbb{R}^{2}$ preserves the (integral) affine structure. However, we can find a map that is an isomorphism almost everywhere.

Indeed, $B-b_{0}$ must be isomorphic to a neighborhood of the puncture in a punctured plane with integral affine structure and monodromy $A$. Specifically, let $X$ be the universal cover of $\mathbb{R}^{2}-0$ with the affine structure lifted from $\mathbb{R}^{2}$ and polar coordinates $(r, \theta),-\infty<\theta<\infty$. With $p=\left(p_{1}, p_{2}\right)$ the Euclidean coordinates on $\mathbb{R}^{2}$, we can also identify points in $X$ by $(p, n)$ where $n=\left[\frac{\theta}{2 \pi}\right]$. Let $V_{n} \subset X, n \geq 1$ be defined by $0<\theta<2 n \pi+\frac{\pi}{2}$. Define the sectors $S_{n}, S_{0} \subset V_{n}$ by $2 n \pi<\theta<2 n \pi+\frac{\pi}{2}$ and $0<\theta<\frac{\pi}{4}$ respectively. Now glue the sector $S_{n}$ to the sector $S_{0}$ via the map that, with respect to the labeling $(p, n)$, sends the point $(p, n)$ to $(A p, 0)$. Call the resulting manifold $P_{n}$.

Lemma 3.4 Each $P_{n}$ defines a Lagrangian fibration $\pi:\left(M_{n}, \omega_{n}\right) \rightarrow P_{n}$ that is unique up to fiberwise symplectomorphism. 
Proof We can construct a Lagrangian torus fibration with base $P_{n}$ as follows: equip $V_{n} \times T^{2}$ with coordinates $(p, q, n)$ and symplectic form $d p \wedge d q$ where $q=\left(q_{1}, q_{2}\right)$ are coordinates on the torus. Now glue $S_{n} \times T^{2}$ to $S_{0} \times T^{2}$ via the symplectomorphism that sends $(p, q, n)$ to $\left(A p, A^{-T} q, 0\right)$. The resulting manifold is $M_{n}$; forgetting the torus coordinates $q$ gives the desired fibration over $P_{n}$. This Lagrangian fibration is uniquely defined by the base because $P_{n}$ has the homotopy type of a 1-dimensional manifold ([4]).

This lemma is clearly still true if we replace $P_{n}$ with a neighborhood $U_{n}$ of the puncture in $P_{n}$. Furthermore, two such neighborhoods $U_{n}, U_{n}^{\prime}$ define a symplectically equivalent Lagrangian fibrations if and only if they are integral affine isomorphic. Note that in terms of the coordinates used in the proof of Lemma 3.4 the vector field $\frac{\partial}{\partial q_{2}}$ on $V_{n} \times T^{2}$ descends to a well defined vector field on $M_{n}$ which for simplicity we also call $\frac{\partial}{\partial q_{2}}$.

Lemma 3.5 Let $\pi:(N, \omega) \rightarrow B$ be a singular Lagrangian fibration with one singular fiber, a nodal fiber with image $b_{0} \in B$ where $B$ is a disk. The punctured disk $B-b_{0}$ is affine isomorphic to a neighborhood of the puncture in $P_{1}$ and $N-\pi^{-1}\left(b_{0}\right)$ symplectically embeds in $M_{1}$ as the preimage of some $U_{1}$. The vanishing cycle of the nodal fiber is the cycle represented by an integral curve of the vector field $\frac{\partial}{\partial q_{2}}$ on $M_{1}$.

Proof One can see that $n=1$ in one of two ways: Duistermaat [4] calculated explicit action coordinates in a neighborhood of a nodal fiber - on the complement of the fibration over a ray based at $b_{0}$. In other words, he found the aforementioned isomorphism. Alternatively, if the boundary of $B$ is chosen to be transverse to rays emanating from $b_{0}$ then the boundary of $N$ is equipped with a contact structure induced from the symplectic structure on $N$. Because this contact structure is fillable, it must be tight (cf. [5]), but this can happen only if $n=1$; otherwise the structure would be overtwisted [8].

The vanishing cycle is in the class of the eigenvector of the monodromy matrix for the torus bundle fibering over $B-b_{0}$. Appealing to the model $M_{1}$ constructed in the proof of Lemma 3.4, we see this is the eigenvector of $A^{-T}$, namely $\frac{\partial}{\partial q_{2}}$.

In $P_{1}$, with coordinates chosen as above, we call the line in the base defined by the vector $(1,0)$ the eigenline. It is the only well defined line that passes through the puncture. 
Two neighborhoods $\left(N_{0}, \omega_{0}\right),\left(N_{1}, \omega_{1}\right)$ of nodal fibers that are Lagrangian fibrations over the same base $B$ need not be fiberwise symplectomorphic. Indeed, there is a Taylor series invariant of the Lagrangian fibration - an element of $\mathbb{R}[[X, Y]]_{0}$, the algebra of formal power series in two variables with vanishing constant term - that classifies such a neighborhood up to fiberwise symplectomorphism [11]. However, we are only interested in classifying the neighborhood up symplectomorphism.

Lemma 3.6 Two neighborhoods $\left(N_{0}, \omega_{0}\right),\left(N_{1}, \omega_{1}\right)$ of nodal fibers that are singular Lagrangian fibrations over the same base $B$ are symplectomorphic.

Proof Let $\mathcal{S}_{0}, \mathcal{S}_{1} \in \mathbb{R}[[X, Y]]_{0}$ be the Taylor series that classify the germs of the neighborhoods of the singular fibers in $N_{0}, N_{1}$. Following San [11], we can use two functions $S_{0}, S_{1} \in \mathcal{C}^{\infty}\left(\mathbb{R}^{2}\right)$ whose Taylor series are $\mathcal{S}_{0}, \mathcal{S}_{1}$ to construct model Lagrangian fibered symplectic neighborhoods equivalent to $N_{0}, N_{1}$. We can choose $S_{0}, S_{1}$ to be equal outside of a small neighborhood $V$ of the origin and then choose a smooth family of functions $S_{t}$ that vanish at the identity, connect $S_{0}$ and $S_{1}$, and are equal to $S_{0}$ and $S_{1}$ outside of $V$. Using these functions we can construct a 1-parameter family of Lagrangian fibered neighborhoods $\left(N_{t}, \omega_{t}\right)$. It is then easy to define a 1-parameter family of diffeomorphisms $\varphi_{t}: N_{0} \rightarrow N_{t}$ such that $\varphi_{0}$ is the identity and $\varphi_{t}^{*} \omega_{t}=\omega_{0}$ on the complement of a smaller neighborhood of the nodal fiber. Because the induced symplectic forms $\varphi_{t}^{*} \omega_{t}$ are all cohomologous a Moser argument completes the proof.

\subsection{Symplectic rational balls}

To prove Theorem 1.3 we need symplectic models for the rational balls $B_{n, m}$ whose boundaries are the lens spaces $L\left(n^{2}, n m-1\right)$. We do this by defining Lagrangian fibrations $\pi:\left(B_{n, m}, \omega_{n, m}\right) \rightarrow U_{n, m}$ with two types of singular fibers: a one parameter family of circle fibers and one nodal fiber.

First note that in our construction of a model neighborhood of a nodal fiber we can make a different choice of coordinates, with respect to which the eigenline is in the $(n, m)$ direction in $\mathbb{R}^{2}$ and the vanishing cycle is in the class of an integral curve of $-m \frac{\partial}{\partial q_{1}}+n \frac{\partial}{\partial q_{2}}$. (Here $m$ and $n$ are relatively prime integers.)

Now let $A_{n, m}$ be a space diffeomorphic to a closed half-plane in $\mathbb{R}^{2}$ and such that: 


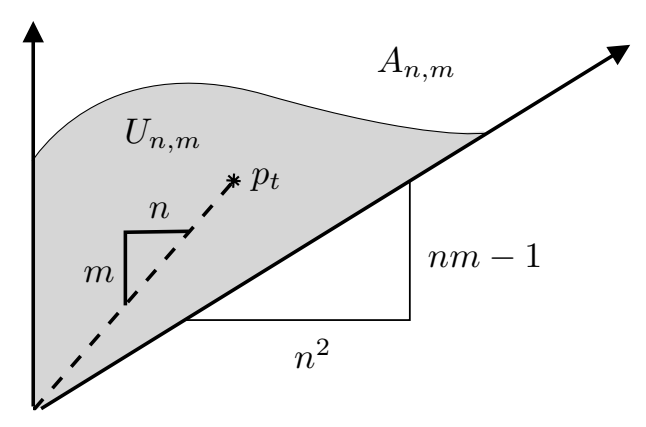

Figure 5: Rational ball with boundary $L\left(n^{2}, n m-1\right)$.

- there is a distinguished point $p_{t} \in \operatorname{int} A_{n, m}$ such that $A_{n, m}-p_{t}$ is equipped with an affine integral structure and the monodromy around $p_{t}$ is $\left(\begin{array}{ll}1 & 1 \\ 0 & 1\end{array}\right)$;

- the eigenline through $p_{t}$ intersects the boundary in a point $p_{0}$; and

- $A_{n, m}$ minus the line segment $L_{t}$ connecting $p_{0}$ and $p_{t}$ is affine isomorphic to the following domain in $\mathbb{R}^{2}$ :

$$
\left\{\left(p_{1}, p_{2}\right) \mid p_{1} \geq 0, p_{2} \geq \frac{n m-1}{n^{2}}, p_{2}>0\right\}
$$

minus the line segment connecting the points $(0,0)$ and $(t n, t m)$ for some $t>0$.

Let $U_{n, m} \subset A_{n, m}$ be a closed neighborhood of $L_{t}$ (which necessarily contains a connected segment of $\left.\partial A_{n, m}\right)$. In Figure 5 we show the image of $U_{n, m}-L_{t} \subset$ $A_{n, m}-L_{t}$ in $\mathbb{R}^{2}$ under the aforementioned isomorphism.

Lemma 3.7 $U_{n, m}$ is the base of a (singular) Lagrangian fibration of the rational ball $B_{n, m}$.

Remark In this description we understand that the preimage of points in $\partial U_{n, m} \cap$ int $A_{n, m}$ are tori so that $U_{n, m}$ defines a manifold with boundary. The image of the boundary is the closure of $\partial U_{n, m} \cap$ int $A_{n, m}$ in $A_{n, m}$.

Proof Because it is homotopic to a 1-manifold, $U_{n, m}-p_{t}$ defines a unique Lagrangian fibration $\pi: M_{0} \rightarrow U_{n, m}-p_{t}$ with $\pi^{-1}(b)$ a circle for each $b \in$ $\partial U_{n, m} \cap \partial A_{n, m}$ (cf. $\left.[2,13]\right)$. 
An open neighborhood of $p_{t} \subset U_{n, m}$ is the base of a singular Lagrangian fibration of a neighborhood of a nodal fiber as in Section 3.2. Therefore we can glue a neighborhood of a nodal fiber into $M_{0}$ with a fiber-preserving symplectomorphism to get a symplectic manifold $M$ fibering over $U_{n, m}$.

To see that $M$ is a rational ball it suffices to note that it is homotopy equivalent to the preimage of an embedded arc connecting the boundary of $U_{n, m}$ and $p_{t}$. This preimage is homeomorphic to the the space obtained from $T^{2} \times[0,1]$ by collapsing all $(1,0)$ curves on $T^{2} \times\{0\}$ (to get the circle fiber over the boundary point) and a $(-m, n)$ curve on $T^{2} \times\{1\}$ (to get the nodal fiber). Because $n \neq 0$, we see $H_{1}(M, \mathbb{R})=H_{2}(M, \mathbb{R})=0$ and $\pi_{1}(M)=\mathbb{Z}_{n}$.

Finally, $M=B_{n, m}$ because its boundary is the lens space $L\left(n^{2}, n m-1\right)$ as can be seen by comparing Figures 3 and 5: a collar neighborhood of the boundary of $M$ projects to a subset of $U_{n, m}$ which is clearly isomorphic to a one sided neighborhood of an arc connecting the two boundary components of $V_{n^{2}, n m-1}$. (See Example 3.1.)

Proposition 3.8 For a given $U_{n, m}$, the rational ball $B_{n, m}$ that fibers over it is unique up to symplectomorphism independent of the choice of $p_{t}$.

For the proof of this we need Zung's classification of integrable Hamiltonian systems with non-degenerate singularities, phrased in terms of Lagrangian fibrations [13]:

Definition 3.9 Two singular Lagrangian fibrations $\rho_{i}:\left(M_{i}, \omega_{i}\right) \rightarrow B_{i}, i=$ 1,2 , are roughly symplectically equivalent if there is an open cover $\left\{U_{\alpha}\right\}$ of $B_{1}$, a homeomorphism $\rho: B_{1} \rightarrow B_{2}$, and fiber preserving symplectomorphisms $\Phi_{\alpha}: \rho_{1}^{-1}\left(U_{\alpha}\right) \rightarrow \rho_{2}^{-1}\left(\phi\left(U_{\alpha}\right)\right)$ such that on $\rho_{1}^{-1}\left(U_{\alpha} \cap U_{\beta}\right)$ the map $\Phi_{\alpha}^{-1} \circ \Phi_{\beta}$ induces the identity map on the fundamental group of the strata of each fiber and the identity map on the first integral homology of each fiber.

Here the fibers are stratified as unions of orbits when one views $\rho_{1}^{-1}\left(U_{\alpha}\right)$ as an integrable Hamiltonian system by composing $\rho_{1}$ with a map $F: U_{\alpha} \rightarrow R^{n}$.

Theorem 3.10 [13] Two singular Lagrangian fibrations that are roughly symplectically equivalent are fiberwise symplectomorphic if and only if they have the same Lagrangian class with respect to a common reference system.

The Lagrangian class of $\pi:(M, \omega) \rightarrow B$ is an element of $H^{1}(B, \mathcal{Z} / \mathcal{R})$ where $\mathcal{Z}$ is the sheaf of local closed 1 -forms $\alpha$ on $M$ such that $\iota_{X} \alpha=\iota_{X} d \alpha$ for any 
vector $X$ such that $\pi_{*} X=0$ and $\mathcal{R}$ is the sheaf of symplectic fiber-preserving $S^{1}$ actions. Identifying a reference Lagrangian fibration is necessary when there is no roughly symplectically equivalent fibration that has a section.

Proof of Proposition 3.8 Cover the base $U_{n, m}$ with a collar neighborhood $V_{b}$ of the boundary and a disk neighborhood $V_{p_{t}}$ of $p_{t}$. Then $V_{b}$ determines a unique Lagrangian fibered manifold [2] and by an isotopy such as in the proof of Lemma 3.6 we can assume that $V_{p_{t}}$ determines a unique Lagrangian fibered manifold. Choosing $\phi$ to be the identity map, the conditions of Definition 3.9 are met because the affine structure on the base determines, up to isomorphism, the sublattice of $H_{1}(F, \mathbb{Z})$ generated by the cycles of a regular fiber $F$ that collapse as the fiber moves to the boundary and to the nodal fiber. Finally, because $H^{1}\left(U_{n, m}, \mathcal{Z} / \mathcal{R}\right)=0$, Theorem 3.10 implies the fibrations are symplectically equivalent [13].

If we vary the position of $p_{t}$ (by varying our choice of $t$ ) we get a family of symplectic forms on the rational ball, all of which are equal near the boundary. Again, the vanishing of the rational cohomology of $B_{n, m}$ allows a Moser argument to confirm that the symplectic structures are isotopic.

The essential element for our proof of Theorem 1.3 is the fact that a collar neighborhood of the boundary of $B_{n, m}$ is well defined up to fiberwise symplectomorphism by its base $V_{b}$.

\section{The symplectic surgery}

With the symplectic models for $B_{n, m}$ and $C_{n, m}$ at hand, the proof of Theorem 1.3 amounts to observing that we can choose $B_{n, m}$ and $C_{n, m}$ so that collar neighborhoods of their boundaries symplectically embed into $L\left(n^{2}, n m-1\right) \times$ $(0, \infty)$, fibering over $V_{n^{2}, n m-1}$ in such a way that their images in $V_{n^{2}, n m-1}$ coincide.

Proof of Theorem 1.3 As explained at the end of Example 3.2, given a symplectic 4-manifold $(M, \omega)$ and an embedding $\psi: C_{n, m} \rightarrow M$ such that each sphere $\psi\left(S_{i}\right)$ is a symplectic submanifold we can assume the embedding $\psi$ is symplectic and gives a Lagrangian fibration $\pi:\left(\psi\left(C_{n, m}\right), \omega\right) \rightarrow W_{n, m} \subset \mathbb{R}^{2}$.

Following Example 3.2 we can choose $u_{0}=(0,-1)$ and $u_{1}=(1,0)$, so the vector $u_{k+1}$ defines a line in $\mathbb{R}^{2}$ with slope $\frac{n m-1}{n^{2}}$. Now $\psi\left(C_{n, m}-\cup_{i=1}^{k} S_{k}\right)$ 
fibers over $W_{n, m}^{\prime}=W_{n, m}-\cup_{i=1}^{k} u_{k}$, so $W_{n, m}^{\prime}$ defines a collar neighborhood of the boundary of $\psi\left(C_{n, m}\right)$. But $W_{n, m}^{\prime}$ can also be viewed as a subset of $A_{n, m}$ so long as the distinguished point $(t n, t m)$ is chosen with $t$ sufficiently small. (See Section 3.3 and Figure 5.) As a subset of $A_{n, m}$ we see that $W_{n, m}^{\prime}$ defines a collar neighborhood of the boundary of a rational ball $B_{n, m}$. Since these two collar neighborhoods fiber over the same simply connected base they are symplectomorphic. Therefore, we can find a symplectomorphism $\phi$ that equips the generalized rational blowdown, $\widetilde{M}=\left(M-\psi\left(\cup_{i=1}^{k} S_{i}\right)\right) \cup_{\phi} B_{n, m}$, with a symplectic structure coming from those on $M$ and $B_{n, m}$.

As for the rational blowdown with $m=1$, the volume of the generalized rational blowdown $\widetilde{M}$ is independent of any choice of rational ball that fits. The argument is exactly the same as in [12]. It would be interesting to know whether a rational blowdown, generalized or not, is unique up to symplectomorphism.

In the above proof we did not mention what is typically a crucial issue when trying to prove a surgery can be done symplectically: symplectic convexity of the neighborhood on which the gluing takes place. A symplectic manifold $(M, \omega)$ with nonempty boundary is symplectically convex if there is an expanding vector field $X$ defined near and transverse to the boundary. To say that $X$ is expanding means $X$ points outward and $\mathcal{L}_{X} \omega=\omega$. The expanding vector field $X$ defines a contact structure on the boundary, the 2-plane field defined as the kernel of the 1 -form $\iota_{X} \omega$ restricted to the boundary.

If $A$ and $B$ are symplectic $2 n$-manifolds with contactomorphic symplectically convex boundaries and $A \subset(M, \omega)$ where $M$ is $2 n$-dimensional, then ( $M-$ int $A) \cup B$ admits a symplectic structure induced from those of $M$ and $B$ (See [5] for more about symplectic convexity, contact structures and symplectic surgeries.)

Thanks to the model spaces, we get symplectic convexity and contactomorphic boundaries for free as follows. Using the same notation as in the proof of Theorem 1.3, we can choose an arbitrarily small Lagrangian fibered neighborhood of spheres $C_{n, m}$ fibering over a $W_{n, m}$ such that the boundary of $W_{n, m}^{\prime}$ is transverse to the vector field $p_{1} \frac{\partial}{\partial p_{1}}+p_{2} \frac{\partial}{\partial p_{2}}$ (when viewed as a subset of $V_{n^{2}, n m-1}$ ). This vector field lifts an expanding vector field on the preimage of $W_{n, m}^{\prime}$, thereby demonstrating the symplectic convexity of $C_{n, m}$. Since we construct $B_{n, m}$ so that a collar neighborhood of its boundary is symplectomorphic to that of $C_{n, m}$, the contact equivalence of the boundaries and the symplectic convexity of $B_{n, m}$ are immediate. 


\section{References}

[1] M. Audin. The topology of torus actions on symplectic manifolds. Birkhäuser Verlag, Basel, 1991.

[2] M. Boucetta and P. Molino. Géométrie globale des systèmes hamiltoniens complètement intégrables: fibrations lagrangiennes singulières et coordonnées action-angle à singularités. C. R. Acad. Sci. Paris Sér. I Math., 308(13):421424, 1989.

[3] A. J. Casson and J. L. Harer. Some homology lens spaces which bound rational homology balls. Pacific J. Math., 96(1):23-36, 1981.

[4] J. J. Duistermaat. On global action-angle coordinates. Comm. Pure Appl. Math., 33(6):687-706, 1980.

[5] J. B. Etnyre. Symplectic convexity in low-dimensional topology. Topology Appl., 88(1-2):3-25, 1998. Symplectic, contact and low-dimensional topology (Athens, GA, 1996).

[6] R. Fintushel and R. J. Stern. Rational blowdowns of smooth 4-manifolds. J. Differential Geom., 46(2):181-235, 1997, alg-geom/9505018.

[7] R. E. Gompf and T. S. Mrowka. Irreducible 4-manifolds need not be complex. Ann. of Math. (2), 138(1):61-111, 1993.

[8] E. Lerman. Contact cuts. To appear in Israel J. Math., math.SG/0002041.

[9] A. McRay. Darboux theorems for pairs of submanifolds. PhD thesis, SUNY Stony Brook, 1994.

[10] J. Park. Seiberg-Witten invariants of generalised rational blow-downs. Bull. Austral. Math. Soc., 56(3):363-384, 1997.

[11] V. N. San. On semi-global invariants for focus-focus singularities. Preprint 524, Institut Fourier, 2001. To appear in Topology.

[12] M. Symington. Symplectic rational blowdowns. J. Differential Geom., 50(3):505-518, 1998, math.DG/9802079.

[13] N. T. Zung. Symplectic topology of integrable Hamiltonian systems, II. Preprint, math.DG/0010181.

[14] N. T. Zung. A note on focus-focus singularities. Differential Geom. Appl., $7(2): 123-130,1997$.

School of Mathematics

Georgia Institute of Technology

Atlanta, GA 30332, USA

Email: msyming@math.gatech.edu

Received: 6 August 2001 Revised: 20 September 2001 